\title{
Non-Mammalian Organisms as Models for
} Cancer

National Cancer Institute

\section{Source}

National Cancer Institute. Non-Mammalian Organisms as Models for Cancer. NCI

Thesaurus. Code C19261.

Studying the origins, progression, and treatment of cancer in a non-mammalian organism 